\title{
Selecting Intrinsic Landmarks in Range Scans
}

\author{
John L. CAMP ${ }^{* a, b}$, Ardeshir GOSHTASBY ${ }^{b}$ \\ ${ }^{a}$ Air Force Research Laboratory, Dayton (OH), USA; \\ ${ }^{b}$ Wright State University, Dayton $(\mathrm{OH})$, USA
}

\begin{abstract}
A method to automatically select landmarks in 3-D range scans is proposed. Selected landmarks represent locally unique intrinsic properties of a scanned surface. The selection process requires no user interaction or surface assumptions. It uses the principal curvatures at the range points to select the landmarks. First, a large number of landmarks are generated by fitting a bicubic polynomial surface to points surrounding each range point and calculating the principal curvatures at the range point. Points of locally extremum principal curvature are then considered candidate landmarks. Candidate landmarks that match with landmarks in other scans of the same subject are then selected as the final, stable landmarks.

Our main goal in landmark selection is to provide a means to characterize models in a range data base. With several scans of each subject available in the data base, a number of stable landmarks are determined for each subject. The locations and characteristics of the landmarks are used to describe a subject and distinguish it from other subjects. The main contribution of this work is considered to be the selection of unique and stable landmarks in a range scan and generation of a descriptor for each landmark that characterizes the intrinsic properties of the surface in the neighborhood of the landmark.
\end{abstract}

Keywords: 3D Models, Landmark Selection, Range Scans, Principal Curvatures, Intrinsic Property

\section{Introduction}

This research develops an automatic method for locating and identifying landmarks in range scans. The identified landmarks are intrinsic to the model subject and are locally unique. The selection process requires no user interaction. This is an important capability providing the means to organize, search, and compare 3-D scanned models. This research offers an efficient means of finding intrinsic landmarks in a range scan by using principal curvature values at range points.

Often, many scans of the same subject are available and there is a need to identify the subject by matching it with known models. Previous studies in automatic landmark detection have used pre-specified locations for detection. This research uses unique and invariant feature characteristics to identify landmarks that characterize the underlying model. The proposed method removes the requirement of having landmarks at fixed locations and allows the method to be applicable to any range scan. Landmarks are selected autonomously by the system from among key feature points that are identified across multiple models of the same object.

The objective of this research is to evaluate the effectiveness of intrinsic landmarks in characterizing models. As a novel approach to model characterization, the utility of intrinsic landmarks is unknown. By developing a method to select intrinsic landmarks and an evaluation technique to determine the validity of the selection, these concerns can be addressed. The objectives of this research are:

1. Given a set of range scans of a subject, select the same landmarks in each scan of the subject.

2. Given a set of range scans of a subject, select a number of stable landmarks specific to the subject and select those same landmarks in future scans of the same subject.

3. Given a set of models, with a set of stable landmarks in each, identify new scans of those models.

4. Given a set of models, with a set of stable landmarks and known characteristics (i.e. gender, body mass index - BMI) in each, predict those characteristics in scans of new models with unknown characteristics.

*camp.6@wright.edu 
Goals 1 and 2 are the problems addressed in this paper. The other goals are applications of the described research.

\section{Literature Review}

Landmark identification in range scans has been the subject of many research programs. Over the past few decades, scanning technology has sufficiently progressed and the availability of high quality range scans has improved. As the quality and availability of range data has increased, so has interest in analyzing and utilizing it. However, this is still a relatively new technology and a fully robust method for identifying landmarks has yet to be developed.

In 1999 the CAESAR (Civilian American and European Surface Anthropometry Resource) project was presented by Robinette et al. [1]. She presented an early overview of the U.S. Air Force Research Laboratory's CAESAR project between 1998 and 2001. Pre-specified landmarks were marked on the human body with physical markers, "flat" white discs when possible and 3-D boxes when the discs would not be visible. There was not a fully automated landmark detection program robust and proven enough to be used; consequently, manual landmark detection was planned. The original plan for detecting landmarks was presented later by Burnsides et al. [2]. Initial efforts showed that manual selection could take up to 60 minutes per scan per subject. With several thousand subjects, the time required to mark the entire data set made a complete implementation unfeasible. This was not only because of the considerable time constraint, but also because of errors introduced when human fatigue sets in after hours of landmark identification. An autonomous system was necessary to fully detect landmarks for thousands of scans.

A method to study range scans was presented by Wang et al $[3,4]$. This method parameterized range data to represent models. Recognizing the difficulty in processing large data sets, the authors devised a 3-step process to generate features of a model. First, a range data set was prepared, then a human model was constructed, and finally, features of the model were extracted. The first step involved noise filtering and model orientation. In order to build the features, a model was first segmented into six parts, the head root, left and right arms, major body, and left and right legs. Each of these segments was cylindrical and was analyzed slice by slice. This resulted in six fully covered segments. Finally, features were extracted based on fuzzy logic using descriptors such as: the chest being about $3 / 4$ of the height from side view. The descriptors were based on garment industry standards and were used to extract dimensions to characterize the segments. The method was efficient and preserved topology; however, in some cases the fuzzy rules selected incorrect body parts. Once the features were parameterized, new models were created without new scans by adjusting the parameters in the existing models.

An approach developed by Leong et al [5] used geometric properties and common proportions to describe a model. This approach did not use markers and identified 21 feature points and 35 feature lines based on geometric properties and common proportions of the human body. Similar to the previous method, a model was segmented into five major parts: arms, legs, and torso-and-head. A range scan was encoded into a 2-D depth map to minimize data size and improve computational speed. The angle and height were used as image coordinates in a 16-bit gray intensity. Laplace and Sobel masks were used to detect features, filtering noise and determining curve properties. The method was successful in detecting feature points and lines in the torso-and-head segment of 5 "standard" size female models.

An additional project reviewed for this study was the work of Ben Azouz et al. [6,7] at the National Research Council of Canada. This research analyzed the CAESAR database mentioned previously. The method required several steps; first step was data processing, which incorporated the following: the surface was smoothed using a Taubin filter, reoriented and segmented to remove the hands and feet, and partitioned into slices. The obtained model was placed in a cube of voxels, each of which was classified as either interior or exterior. The model was then converted to a vector with deviation vectors representing the difference in distances from the average model. Eigenvectors of the matrix representing these deviation vectors were used to define the model; with the first 40 eigenvectors spanning $92 \%$ of the variability. Each principal component was highly correlated to a body shape description. 
This research was later expanded by Ben Azouz et al. [8,9]. Using the volumetric representation and the eigenvectors, it was demonstrated that model variation could be created by altering the principal components. Furthermore, a landmark detection method was implemented by inferencing over a pair-wise Markov random field. SPIN images [10] were used to characterize local surface features which were then matched to a best fit in the Markov random field. 200 models were used to learn an initial loop belief. 30 additional models were used to validate the method, obtaining encouraging results.

\section{Proposed Approach}

With the increasing availability of range scans of the human body, it is critical to develop a method for sorting and comparing the scans. Existing methods have limitations. The methodology presented here identifies unique landmarks in the scans. Landmark selection is achieved by analyzing every range point and calculating its principal curvatures and directions, and determining the average curvature of points surrounding each landmark. Feature points are selected based on the local strength of these features. As new scans of the same subject become available, the same intrinsic landmarks are identified in them.

\subsection{Intrinsic Landmarks}

Landmarks are invariant feature points on a model. Given multiple scans of various objects of the same type (i.e. - humans, cars, cows, etc.), each object can be recognized by a set of unique landmarks. These landmarks characterize the local geometry of the surface of the object. The landmarks can define an object by their number, locations, and characteristics.

\subsection{Landmark Characterization}

The identification and characterization of landmarks in a range scan is a non-trivial task. It is possible to visually locate landmarks on a model based on pre-specified characteristics. However, when there are numerous landmarks to find and there are thousands of models, the problem becomes intractable, due to time and accuracy constraints. It is also possible to pre-mark desired landmarks on a subject prior to scanning. However, this is also time-consuming and is prone to user bias. These limitations can be overcome by designing a method that can automatically select landmarks on range data that are intrinsic to the model.

In order to automatically select landmarks, it is necessary to establish a consistent method of characterization. The proposed method examines each range point and considers its surrounding region. The critical values considered are the maximum and minimum principal curvatures. Given a three dimensional surface, at any point, there are two orthogonal principal curvature directions. The maximum and minimum curvature directions indicate the direction of the greatest and least curvature, respectively. Both directions lie on a plane tangent to the surface at the point and are, therefore, orthogonal to the surface normal. The magnitude of the curvature value represents how curved the patch is along that curvature direction. Consider the patch shown in Figure 1. The middle image displays the patch as seen down the direction of minimum curvature; the direction of maximum curvature can be seen originating at the center of the image and continuing off to the left. Likewise, the image to the right shows the patch as seen down the direction of maximum curvature; the direction of minimum curvature can be seen originating at the center of the image continuing off to the right. It is important to note that the principal curvature directions represent the direction of maximum and minimum curvature at that point on the surface; they are not curvature contour lines which represent continuous curves of maximum or minimum curvature along the patch.
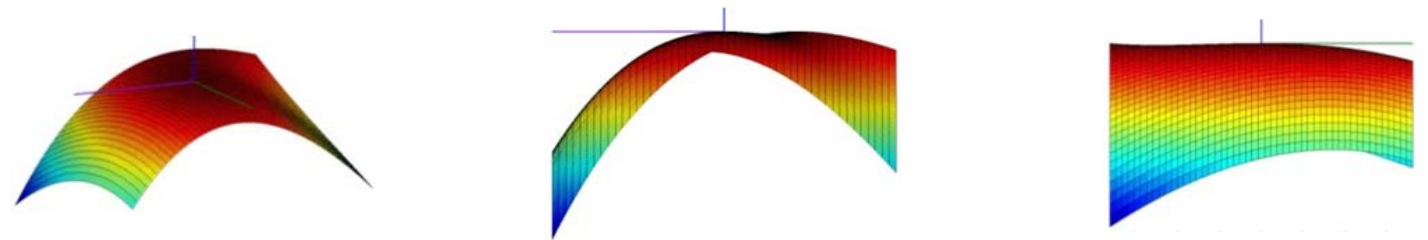

Fig.1. Left, the patch is shown from an arbitrary view angle. Middle, the patch is shown from a view down the direction of minimum curvature. Right, the patch is shown from a view down the direction of maximum curvature. 
Once the principal curvatures and directions for every point are calculated, each point can be characterized based on their own curvatures, as well as the curvatures of the points surrounding it. The neighborhood of points is characterized by the surface normal and principal curvature directions, which are aligned with the surface normal along the $z$-axis, the direction of maximum curvature along the $x$-axis and the direction of minimum curvature along the $y$-axis. This allows the characterization to be invariant to model orientation. Regardless of the orientation of the point on the model, the direction of maximum curvature will be consistent. The points in a neighborhood are grouped into one of 16 bins, which subdivide the region immediately surrounding the current point into equal size regions. With this arrangement, each point is characterized based on 34 values, which include its own maximum and minimum principal curvature values as well as the average maximum and minimum principal curvature values of the points for each of the 16 bins. This characterization describes not only the attributes of the point itself, but that of the region surrounding it as well. In this way, landmarks are distinctively described.

\subsection{Characterizing a Landmark and its Neighborhood}

To characterize a landmark, principal curvatures are calculated from an explicit function defining the surface surrounding the landmark. However model data is not stored as a smooth surface. In fact, the scanners generally generate a point cloud that represents the surface of the subject. A computer program generates polygons (usually triangles) to connect the points and approximate a range scan to a model by a polygon mesh. The point and polygon representation of a subject is an efficient method for data storage and provides an approximation to the model for most uses. Certainly, a single explicit function cannot be used to accurately define the shape of the subject. Nevertheless, an explicit function is necessary and can instead be calculated locally for each point. By examining all the connected points within a specified distance of the current point, a surface can be fitted to them to define and explicit surface. This surface will accurately represent the smooth shape surrounding the point and provide accurate principal curvature data for landmark characterization. Figure 2 displays the fitting of a surface to a landmark and range points in its neighborhood.
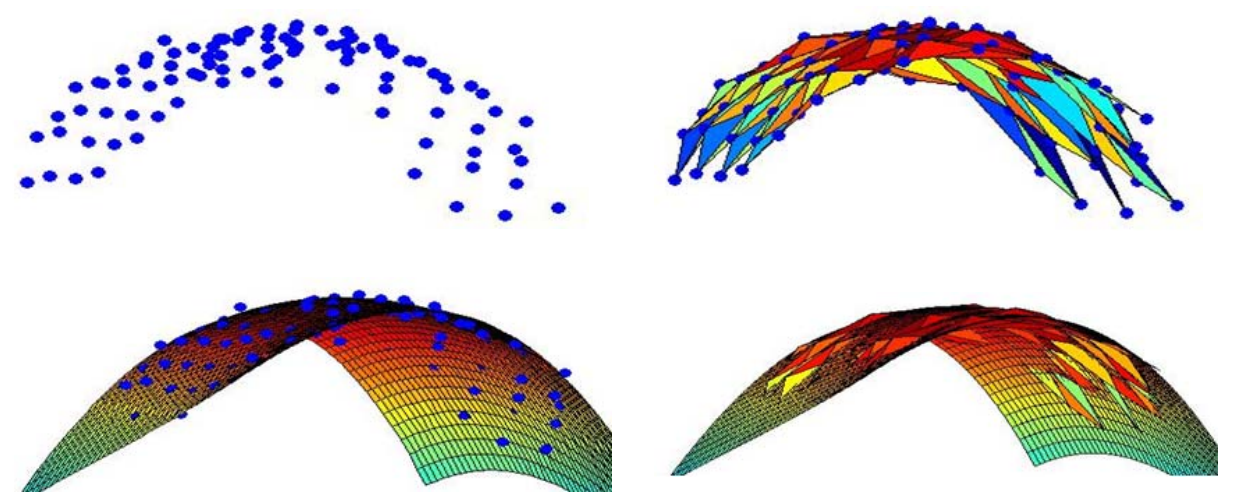

Fig 2. A local neighborhood, clockwise from the top left: the points, the points after triangulation, the surface and the triangular mesh, and finally, the points and the fitted surface.

\section{Implementation}

With an established landmark characterization method, a methodology is implemented to automatically select landmarks in a data set consisting of points and a triangle mesh. Incorporating an acceptable set of practical assumptions and constraints, a range scan is preprocessed prior to automatic landmark selection. The implemented methodology consists the following steps:

1. Identifying the neighborhood of the point currently under consideration.

2. Fitting a bi-cubic surface to the neighborhood.

3. Characterizing the point based on 34 total principal curvature values, including the maximum and minimum principal curvature of the landmark and its 16 surrounding regions.

4. Selecting feature points based on the local strength of each point's principal curvature values.

5. Identifying intrinsic landmarks based on a random sample and consensus (RANSAC) algorithm that matches feature points within the subject's scans. 


\subsection{Neighborhood Establishment}

The first step of the landmark selection process is to select the neighborhood of a point. Using the mesh data, it is possible to select only connected points within a given radius, avoiding those points that are close but not connected to the point under consideration within the specified radius.

After selecting the neighborhood, the points within the neighborhood are translated so that the current point moves to the origin. Because the principal curvature values are based only on local shape, the neighborhood can be examined in isolation from the rest of the model. This removes the impact of a model's position and orientation. The neighborhood is oriented such that the surface normal is along the $z$-axis, placing the principal curvature directions in the $x-y$ plane. This is accomplished by approximating the surface normal by fitting a plane through the current point and minimizing the distance of each point in the neighborhood to the plane using a linear least squares algorithm. The plane normal is used to approximate the surface normal and the entire neighborhood is rotated so that the plane normal is along the $z$-axis.

\subsection{Surface Fitting}

After the neighborhood has been properly oriented, an explicit function is fitted to the points. The explicit function is defined by the following bi-cubic polynomial:

$$
f(x, y)=\sum_{i=0}^{3} \sum_{j=0}^{3} a_{i j} x^{i} y^{j} .
$$

The coefficients of the polynomial are determined using a weighted least squares algorithm to minimize the weighted sum or square distances between the calculated $f(x, y)$ and the $z$ values of points in the neighborhood, with the points closer to the origin given a greater weight. Given this definition of the surface, the actual surface normal direction can be computed. The neighborhood and surface are rotated such that the true surface normal is along the $z$-axis.

With the explicit function defining the surface locally, the principal curvatures and directions are determined directly as follows [10]. These equations are based on the first and second fundamental forms of a surface. The forms require a parameterization of the surface. Using the following parameterization:

$$
\mathbf{p}(u, w)=[u, w, f(u, w)],
$$

where, $u$ corresponds to $x$ and $w$ to $y$. The first form is given by,

$$
d \mathbf{p} \cdot d \mathbf{p}=E d u^{2}+2 F d u d w+G d w^{2} .
$$

The coefficients are calculated as follows:

Likewise, the second form is given by,

$$
\begin{aligned}
& E=\boldsymbol{p}^{u} \cdot \boldsymbol{p}^{u} \\
& F=\boldsymbol{p}^{u} \cdot \boldsymbol{p}^{w} \\
& G=\boldsymbol{p}^{w} \cdot \boldsymbol{p}^{w}
\end{aligned}
$$

$$
-d \mathbf{p}(u, w) \cdot d \mathbf{n}(u, w)=L d u^{2}+2 M d u d w+N d w^{2} .
$$

The coefficients are calculated as follows:

$$
\begin{aligned}
& L=\boldsymbol{p}^{u u} \cdot \mathbf{n}, \\
& M=\boldsymbol{p}^{u w} \cdot \mathbf{n}, \\
& N=\boldsymbol{p}^{w w} \cdot \mathbf{n} .
\end{aligned}
$$

With these coefficients, the principal curvatures can be calculated by solving the following equation:

$$
\left(E G-F^{2}\right) \varkappa^{2}-(E N+G L-2 F M) \mathcal{H}+\left(L N-M^{2}\right)=0 .
$$


The roots of this equation are the principal curvature values. The principal directions can be found, by considering:

$$
h=d w / d u,
$$

and solving the following quadratic equation:

$$
(F N-G M) h^{2}+(E N-G L) h+(E M-F L)=0 .
$$

Here, roots, $h_{1}$ and $h_{2}$ represent the ratio in the $d w / d u$ (or $y / x$ ) plane between the curvature directions. So, the curvature directions are:

$$
\begin{aligned}
& \operatorname{curvDir}_{1}=\left[h_{1}, 1,0\right], \\
& \operatorname{curvDir}_{2}=\left[h_{2}, 1,0\right] .
\end{aligned}
$$

After determining the principal curvature directions, the neighborhood must be reoriented so that the maximum curvature direction is on the $x$-axis and the minimum curvature direction is on the $y$-axis. By aligning the principal curvature directions to the $x$ and $y$ axes, the shape (based on curvature values) of the region surrounding the point can be consistently compared to the shape of other points. Since the neighborhood is examined in isolation from the model, this alignment assures that there is uniformity in the orientation of neighborhoods under consideration. The principal curvature directions found are not explicitly associated with the maximum or minimum direction. The proper association is determined by checking every point in the neighborhood to find a point closest to the positive and negative axis of each curvature direction. Based on the average slope of change for the $z$ value of the two points on each axis, the maximum curvature direction is selected as the axis with the largest $z$ slope, representing the greatest curve.

Finally, each point in the neighborhood is associated with one of the bins subdividing the region. The neighborhood is divided around the current point (at the origin) into 16 equal size, square bins, via a $4 \times 4$ grid in the $x / y$ plane.

\subsection{Feature Point Selection}

Feature points are selected by examining each point. The maximum principle curvature value of that point is checked against the maximum principle curvature values of its neighbors. The examined point is marked as a local maximum, local minimum, or neither for the maximum principle curvature. Likewise, the examined point is labeled based on its minimum principle curvature value. Finally, the examined point is considered to be a feature point if its maximum principle curvature value is locally maximum and its minimum principle curvature value is a either locally maximum or minimum.

\subsection{Point Characterization}

After calculating the principal curvature values and surface normal direction for every point in the model, each point can be fully characterized. For each of the sixteen bins surrounding the point, the average maximum and minimum principal curvature values are calculated within each bin and stored in an array. Each point is characterized by this array, along with its own maximum and minimum principal curvature values. The process efficiently describes the local model shape around the point.

\subsection{Intrinsic Landmark Selection}

The selection of the intrinsic landmarks is accomplished by examining several models of the same subject. For each model, feature points are generated independently. A RANSAC [12] method is utilized to define the transformation between any two of the models, using the matching feature points. First, a set of four feature points are selected from one of the models. Four points are the required to determine the 12 unknown coefficients of the affine transformation defined by:

$$
\begin{aligned}
& X_{i}=a x_{i}+b y_{i}+c z_{i}+d \\
& Y_{i}=e x_{i}+f y_{i}+g z_{i}+h \\
& Z_{i}=j x_{i}+k y_{i}+l z_{i}+m
\end{aligned}
$$


These selected points, with their coordinates: $\left(x_{i}, y_{i}, z_{i}\right)$, are matched to feature points in another model, with coordinates: $\left(X_{i}, Y_{i}, Z_{i}\right)$. This match is made based on the similarity of the 34 curvature characteristics of the two points. Next, the coefficients of the affine transformation are calculated mapping the coordinates of the second model to that of the first by linear least squares. Using these equations, the remaining, unmatched feature points of the second model are transformed to the coordinate system of the first. Then, for each transformed feature point, a check is made to determine whether there is a feature point within its distance $\varepsilon$. When that is the case, the points are considered matching. If at least $85 \%$ of the feature points match, then the RANSAC is considered complete. However, if there isn't enough matching feature points, the previous steps are repeated, with a new set of 4 points. This is continued until at least $85 \%$ of the feature points match.

After establishing correspondence between feature points in two models, intrinsic landmarks are selected from the feature points. That involves finding feature points that have matches across all the models of the subject. This process identifies stable feature points that appear in all models.

\section{Results}

The proposed method, as implemented, is generally capable of selecting intrinsic landmarks from point cloud and triangular mesh data sets containing multiple scans of a subject. In order to evaluate the effectiveness of the method, a data set generated from the Air Force Research Laboratory is used. This data set consists of twenty subjects, male and female, in three poses, each pose scanned three times in two different scanners. For this initial evaluation, each of the six scans from one pose, for all twenty subjects is considered.

Table 1. Landmark selection results showing average deviation from mean of landmarks, after affine matching.

\begin{tabular}{|c|c|c|}
\hline Model & $\begin{array}{c}\text { Average Deviation } \\
\text { from Mean }(\mathrm{mm})\end{array}$ & $\begin{array}{c}\text { Standard Deviation of } \\
\text { Deviation from Mean } \\
(\mathrm{mm})\end{array}$ \\
\hline 1 & 7.80754 & 0.01463 \\
\hline 2 & 7.48236 & 0.01436 \\
\hline 3 & 7.70167 & 0.01408 \\
\hline 4 & 7.56837 & 0.01372 \\
\hline 5 & 7.55513 & 0.01355 \\
\hline 6 & 7.66257 & 0.01408 \\
\hline 7 & 7.70397 & 0.01492 \\
\hline 8 & 7.50887 & 0.01435 \\
\hline 9 & 7.74309 & 0.01415 \\
\hline 10 & 8.04403 & 0.01444 \\
\hline 11 & 7.52169 & 0.01371 \\
\hline 12 & 7.63578 & 0.01406 \\
\hline 13 & 7.56136 & 0.01421 \\
\hline 14 & 7.38253 & 0.01361 \\
\hline 15 & 7.83911 & 0.01478 \\
\hline 16 & 7.69913 & 0.01357 \\
\hline 17 & 7.54301 & 0.01353 \\
\hline 18 & 7.64389 & 0.01446 \\
\hline 19 & 7.44141 & 0.01394 \\
\hline 20 & 7.59805 & 0.01402 \\
\hline Average & 7.63218 & 0.01411 \\
\hline & & \\
\hline
\end{tabular}


Intrinsic landmarks were selected for twenty subjects based on the feature points generated in the six scans from the same pose using the following RANSAC criteria: start with 4 matching points, use matching tolerance, $\varepsilon$, of $3.5 \mathrm{~mm}$, and stop RANSAC when $85 \%$ of points match. The results are given in Table 1. This table shows how closely the matched landmarks are clustered when transformed to the coordinate system of one model. The mean deviation for each model represents the average distances calculated for each selected landmark in that model. This distance is measured by calculating the centeroid of the six matched landmarks, then averaging the Euclidian distance between the centeroid and each matched landmark. So, for Model 1, the mean deviation of each matched landmark from its cluster centeroid is $7.80 \mathrm{~mm}$, with a standard deviation of $0.01 \mathrm{~mm}$. Overall, the mean deviation of matched landmarks from cluster centeroids was less than $8 \mathrm{~mm}$, with a standard deviation of $0.01 \mathrm{~mm}$. A set of selected landmarks can be seen in Figure 3, which displays landmarks selected in six scans of one subject.
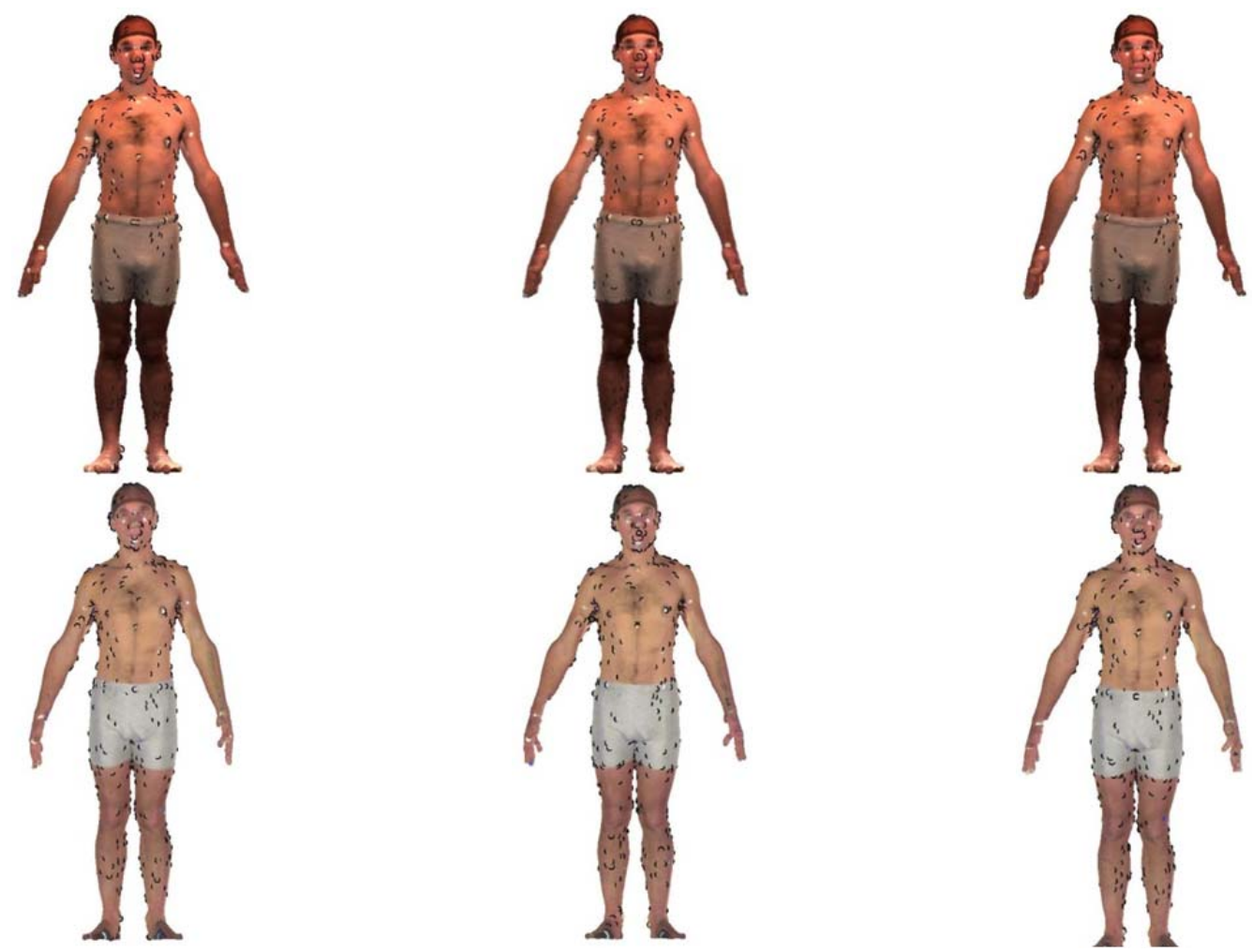

Fig.3. Automatically selected intrinsic landmarks of a subject, where the black dots mark selected landmarks, found in all six scans. The white dots are clothing industry standard landmarks, physically marked on the subjects prior to scanning.

\section{Future Work}

The core method has been implemented with encouraging results; this completes Goals 1 and 2, which were to select landmarks across all scans of a subject and identify the stable landmarks in additional scans. The significant step in the research is to address Goal 3, the identification of new scans of known subjects and Goal 4, which is to predict characteristics of unknown subjects.

\section{Summary and Conclusions}

A method for selecting intrinsic landmarks was introduced demonstrating encouraging results. Currently, the method can select these landmarks within mean deviation of about $8 \mathrm{~mm}$. This is a useful capability that can be used to characterize and match any 3-D model set that is stored as a triangular mesh. The proposed method does not require any subject markings, specified landmark definitions, or user input; it is a fully autonomous system. Future work will demonstrate its ability in model identification and matching. 


\section{References}

1. K. M. Robinette, H. Daanen, and E. Paquet, "The CAESAR Project: A 3-D Surface Anthropometry Survey," 1999.

2. D. Burnsides, M. Boehmer, and K. Robinette, "3-D Landmark Detection and Identification in the CAESAR Project," 2001.

3. C. C. L. Wang, "Parameterization and Parametric Design of Mannequins," Computer-Aided Design 37, 2005.

4. C. C. L. Wang, T. K. K. Chang, and M. M. F. Yuen, "From laser-scanned data to feautre Human Model: A System Based on Fuzzy Logic Concept," Computer-Aided Design 35, 2003.

5. I.-F. Leong, J.-J. Fang, and M.-J. Tsai, "Automatic Body Feature Extraction from a Marker-Less Scanned Human Body," Computer Aided Design 39, 2007.

6. Z. Ben Azouz, M. Rioux, C. Shu, and R. Lepage, "Analysis of Human Shape Variation Using Volumetric Techniques," The 17th Annual Conference on Computer Animation and Social Agents, Jul. 2004.

7. Z. Ben Azouz, M. Rioux, C. Shu, and R. Lepage, "Characterizing Human Shape Variation Using 3-D Anthropometric Data," The Visual Computer International Journal of Computer Graphics, vol. 22, no. 5, pp. 302-314, 2005.

8. Z. Ben Azouz, C. Shu, and A. Mantel, "Automatic Locating of Anthropometric Landmarks on 3D Human Models," Third International Symposium on 3D Data Processing, Visualization and Transmission, Jun. 2006.

9. Z. Ben Azouz, C. Shu, R. Lepage, and M. Rioux, "Extracting main Modes of Human Shape Variation from 3-D Anthropometric Data," Fifth International Conference on 3-D Digital Imaging and Modeling, Jun. 2005.

10. A. E. Johnson and M. Hebert, "Recognizing Objects by Matching Oriented Points, Proc.," Computer Vision and Pattern Recognition, pp. 684-689, 1997.

11. M. E. Mortenson, Geometric Modeling, Third Edition, New York, New York: Industrial Press Inc., 2006.

12. R. Hartley and A. Zisserman, Multiple View Geometry in Computer Vision, Second Edition, Cambridge University Press, pp 117-121, 2003. 